$$
\begin{aligned}
& 1 N-08 \\
& 081470
\end{aligned}
$$

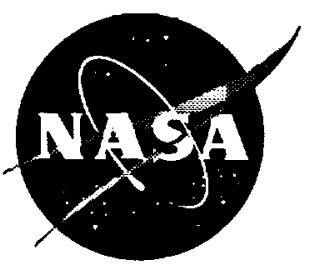

\title{
Wavelet Filtering to Reduce Conservatism in Aeroservoelastic Robust Stability Margins
}

Marty Brenner

Dryden Flight Research Center

Edwards, California

Rick Lind

National Research Council

NASA Dryden Research Associate

Dryden Flight Research Center

Edwards, California

National Aeronautics and

Space Administration

Dryden Flight Research Center

Edwards, California 93523-0273 


\section{NOTICE}

Use of trade names or names of manufacturers in this document does not constitute an official endorsement of such products or manufacturers, either expressed or implied, by the National Aeronautics and Space Administration.

\section{Available from:}

NASA Center for AeroSpace Information 800 Elkridge Landing Road Linthicum Heights, MD 21090-2934

Price Code: A16
National Technical Information Service 5285 Port Royal Road Springfield, VA 22161 Price Code: A16 


\title{
WAVELET FILTERING TO REDUCE CONSERVATISM IN AEROSERVOELASTIC ROBUST STABILITY MARGINS
}

\author{
Marty Brenner* \\ Aerostructures Branch \\ Rick Lind ${ }^{\dagger}$ \\ NASA Dryden Flight Research Center
}

\begin{abstract}
Wavelet analysis for filtering and system identification was used to improve the estimation of aeroservoelastic stability margins. The conservatism of the robust stability margins was reduced with parametric and nonparametric timefrequency analysis of flight data in the model validation process. Nonparametric wavelet processing of data was used to reduce the effects of external disturbances and unmodeled dynamics. Parametric estimates of modal stability were also extracted using the wavelet transform. Computation of robust stability margins for stability boundary prediction depends on uncertainty descriptions derived from the data for model validation. F-18 High Alpha Research Vehicle aeroservoelastic flight test data demonstrated improved robust stability prediction by extension of the stability boundary beyond the flight regime
\end{abstract}

\section{Nomenclature}

$\begin{array}{ll}a, a_{i} & \text { wavelet scale, indexed scale values } \\ A & \text { residue magnitude } \\ \text { ASE } & \text { aeroservoelastic } \\ \text { DWT } & \text { discrete wavelet transform } \\ \text { FFT } & \text { fast Fourier transform } \\ F(P, \Delta) & \text { feedback interconnection structure } \\ g & \text { wavelet basis function } \\ G, \alpha, \tau & \text { spectrum of wavelet } g \\ \text { HARV } & \text { high-alpha research vehicle } \\ i & \text { imaginary unit } \\ k(t) & \text { envelope signal of } x(t) \\ K & \text { feedback control system }\end{array}$

\footnotetext{
-Aerospace Engineer, 805.258.3793, martin.brenner@dfrc.nasa.gov, Member AIAA

†NRC PostDoctoral Research Fellow, 805.258.3075, rick.lind@dfrc.nasa.gov, Member AIAA

Copyright $(\mathcal{C} 1998$ by the American Institute of Aeronautics and Astronautics, Inc. No copyright is asserted in the United States under Title 17, U.S. Code. The U.S. Government has a royalty-free license to exercise all rights under the copyright claimed herein for government purposes. All other rights are reserved by the copyright owner.
}

\begin{tabular}{|c|c|}
\hline OBES & on-board excitation system \\
\hline$P(s)$ & Laplace transform of system plant \\
\hline$\hat{P}(s)$ & Laplace transform of system plant estimate \\
\hline $\bar{q}$ & dynamic pressure \\
\hline$s$ & Laplace frequency \\
\hline$t$ & time \\
\hline$u$ & system input \\
\hline WT & wavelet transform \\
\hline$W_{g}$ & continuous WT with basis $g$ \\
\hline$W_{\text {add }}$ & weighting on $\Delta_{a d d}$ \\
\hline$W_{i n}$ & weighting on $\Delta_{i n}$ \\
\hline$W_{n s}$ & weighting on noise \\
\hline$x(t)$ & time domain signal \\
\hline$\hat{x}(t)$ & filtered time domain signal \\
\hline$X(\omega)$ & frequency domain signal \\
\hline$\hat{X}(\omega)$ & estimate of frequency domain signal \\
\hline$X(\tau, \omega)$ & wavelet-transformed signal \\
\hline$\hat{X}(\tau, \omega)$ & filtered wavelet-transformed signal \\
\hline$y$ & system output \\
\hline$\alpha$ & angle of attack \\
\hline$\gamma$ & frequency step constant \\
\hline$\Gamma$ & robust stability margin \\
\hline$\delta_{\bar{q}}$ & uncertainty in flight condition \\
\hline$\Delta$ & uncertainty operator \\
\hline$\hat{\Delta}$ & uncertainty operator estimate \\
\hline$\Delta_{a d d}$ & uncertainty at output from plant errors \\
\hline$\Delta_{i n}$ & input uncertainty \\
\hline$\Delta_{A}$ & modal parametric uncertainty \\
\hline$\zeta$ & damping ratio \\
\hline$\mu$ & structured singular value \\
\hline$\tau$ & wavelet translation time \\
\hline$\phi(t)$ & signal phase \\
\hline$\phi_{o}$ & constant phase lag \\
\hline$\omega$ & radian frequency \\
\hline$\omega_{d}$ & modal damped frequency \\
\hline$\omega_{n}$ & modal natural frequency \\
\hline$\omega_{o}$ & wavelet peak frequency \\
\hline * & complex conjugate \\
\hline$L$ & phase angle \\
\hline 11 & modulus \\
\hline\|\|$_{\infty}$ & operator infinity norm \\
\hline
\end{tabular}




\section{Introduction}

Envelope expansion of new or modified aircraft often requires structural stability testing to verify safety margins to prevent against aeroservoelastic (ASE) instability. Inflight testing allows determination of aeroelastic or ASE effects as a function of flight parameters. Flight data is acquired for stability estimation and system identification to compare with analytic predictions. Any anomalies are regarded with care for safety of flight.

Excitation systems are often essential to establish stability trends from noisy measurements since atmospheric turbulence is generally insufficient to provide adequate levels of excitation ${ }^{3}$. These systems often generate deterministic nonstationary input signals. Wavelet signal processing has shown promise for system identification in such environments by application as filter banks for data enhancement. Improvement in flight data analysis is achieved by discriminating areas of low signal-tonoise ratio, unmodeled dynamics, and external disturbances. Removing aspects of signal responses detrimental to linear identification methods may improve stability tracking ${ }^{2,5,21}$.

Wavelet transforms have also been applied to parametric identification of time-varying multiple degree-of-freedom systems by estimating the impulse response using correlation methods ${ }^{6,9}$. Modal frequency and damping parameters are estimated directly from the data without intermediate model identification schemes. In these schemes, parameter range approximations are necessary to discriminate frequency and damping.

A recent method $^{18}$ uses a wavelet transform (WT) on free response data to directly supply information on time-dependent modal decay rate and phase variation. Without any approximation of parameter range, natural frequencies and damping ratios are extracted from the response. Damping and frequency trends are useful for noting changes in system dynamics as a function of flight condition, thereby helping to reduce conservatism in real parameter variations of the uncertainty model.

Model validation is a critical procedure in the computation of robust stability margins. The margins are adversely affected by poor characterizations of the uncertainty size and structure, which are determined by perturbation magnitude, location in the system, and type (real or complex). Wavelet processing of ASE flight test data improves the robust stability margin estimate by helping to reduce the conservatism in the uncertainty description pertaining to both complex (nonparametic) and real (parametric) perturbations.

This paper augments wavelet filtering with waveletbased modal parameter extraction to produce robust stability margins with reduced-norm uncertainty sets of both complex-nonparametric and real-parametric peturbations. The decrease in conservatism results in a more practical and valuable robust stability margin.

Transfer functions and modal parameter estimates derived from time-frequency Morlet wavelets are used to estimate state space ASE models from F-18 $\mathrm{HARV}^{1}$ (High Alpha Research Vehicle, fig. 1) flight data. These models are used in a robust stability boundary prediction method based on the structured singular value, $\mu^{10,11}$.

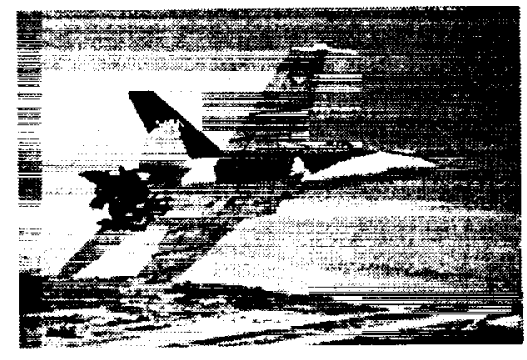

Figure 1: F-18 HARV

\section{F-18 HARV and ASE Flight Test}

The F-18 HARV aircraft is a two-seat fighter that was modified to include thrust vectoring paddles on the engines and a research flight control system to ensure stability at high angle-of-attack flight conditions ${ }^{17}$. The flight system also included an excitation signal generator, designated as OBES (on-board excitation system), for aerodynamic parameter identification, closed-loop stability monitoring, and aeroservoelastic excitation ${ }^{4}$. For ASE stability monitoring, the OBES was configured to sum programmed digital signals to the control system actuator commands for structural excitation of the primary modes (table 1). Inputs from 5 to $20 \mathrm{~Hz}$ were added to the control surface commands at angles of attack from 5 to $70 \operatorname{deg} \alpha$ at $\mathrm{lg}$.

\begin{tabular}{cc}
\hline \hline Antisymmetric Mode & $\omega(\mathrm{Hz})$ \\
\hline Fuselage $1^{\text {st }}$ Bending & 7.1 \\
Wing $1^{\text {st }}$ Bending & 8.8 \\
Wing $1^{\text {st }}$ Torsion & 12.0 \\
Stabilizer $1^{\text {st }}$ Bending & 13.6 \\
Wing Fore-Aft & 15.2 \\
Fin $1^{\text {st }}$ Bending & 15.7 \\
Fuselage $1^{\text {st }}$ Torsion & 19.1 \\
Fuselage $2^{\text {nd }}$ Bending & 21.4 \\
Exhaust Vane Rotation & 22.1 \\
Inboard Flap Rotation & 23.2 \\
Fore-Fuselage Torsion & 24.2 \\
\hline \hline
\end{tabular}

Table 1: F-18 HARV calculated elastic frequencies. 
Analytical predictions indicated poor ASE stability robustness in the lateral-directional feedback loops. Structured singular values of complementary sensitivity near the first antisymmetric wing bending and wing torsion modes (about $9 \mathrm{~Hz}$ and $12 \mathrm{~Hz}$, respectively) approached $0 \mathrm{~dB}$, and the wing-fore aft mode near $15 \mathrm{~Hz}$ was at $-6 \mathrm{~dB}$. Flight envelope limits were 15,000 to $35,0000 \mathrm{ft}$ altitude up to Mach 0.7. Worst-case flight conditions from the analysis were less than Mach 0.3 , greater than 30,000 ft altitude, and above $50 \operatorname{deg} \alpha$. This paper addresses robust stability at a representative worst-case flight condition of $50 \mathrm{deg} \alpha$ at Mach 0.3 and altitude 30,000 ft.

\section{Time-Frequency System Identification}

A desirable feature of signal analysis is adaptation to both transient and stationary characteristics, which implies both time and frequency domain resolution criteria subject to the uncertainty principle. These competing requirements demand a method that is tunable according to the local signal dynamics. For general types of input excitation, constant time-frequency resolution analysis ${ }^{2,5}$ may not be applicable.

Redundant continuous wavelet transform methods give arbtrarily good resolutions, but are cumbersome ${ }^{7}$ and often slow ${ }^{13}$ for reconstruction and filtering. Alternatively, non-redundant (compact and orthonormal) wavelet transforms are fast and accurate, but are limited in frequency resolution even with wavelet packets. Good frequency resolution is obtained with classical harmonic wavelets ${ }^{15}$, but time resolution is sacrificed. The objective of adjusting the competing requirements of time and frequency resolution with fast, accurate processing is accomplished with a combination of compact orthogonal and harmonic wavelet properties in the compact harmonic wavelets ${ }^{7,8}$.

\section{Nonparametric Estimation - Wavelet Filtering}

The multivoice wavelet transform was introduced to exploit multiresolution analysis using compact harmonic wavelets ${ }^{7,20}$. Multivoice, or multiscale, refers to redundant representations of signals on multiple frequency bands ${ }^{19}$. Nonorthonormal Morlet wavelets are approximated with (harmonic-like) discretizations on multiple wavelet scales. These form a nonorthogonal redundant basis for the signal space, which does not admit a multiresolution analysis. The discrete wavelet transform (DWT) needs to be derived from the wavelet basis to get a multiresolution analysis of the sampled continuous Morlet transform ${ }^{20}$.

The DWT is implemented as a filter bank covering a predefined range of frequencies with corresponding number of frequency bands (voices) per octave. Interpolation, or scaling, filters are introduced to define how the scales relate to each other in a dyadic fashion for the multiscale representation. These scaling filters are compact (finite impulse response) for fast and accurate reconstruction. Therefore, multivoice transforms provide practical, fast, and flexible means for analysis and filtering of nonstationary data with tunable frequency resolution versus time localization.

The wavelet transform of signal $x(t)$ over the time-scale $(\tau, a)$ plane is represented as

$$
W_{g}(\tau, a)=\frac{1}{\sqrt{a}} \int x(t) g^{*}\left(\frac{t-\tau}{a}\right) d t
$$

where scale parameter $a$ is proportional to the duration and inversely proportional to the peak frequency $\omega_{o}$ of the complex Morlet wavelet

$$
g(t)=\frac{1}{\sqrt{2 \pi}} e^{-\frac{z^{2}}{2}} e^{i \omega_{0} t} .
$$

The spectrum of a dilated and translated Morlet wavelet

$$
G_{a, \tau}(\omega)=e^{-\left(a \omega-\omega_{0}\right)^{2}} e^{i \omega \tau}
$$

reaches a maximum value at $a=\frac{\omega_{0}}{\omega}$. Frequency discretization is logarithmic in the frequency range of interest by setting the sequence of scale values to $a_{i}=a_{0} \gamma_{i}$, where $(\log \gamma, \gamma>1)$, is the constant frequency step. Integration step $\log \gamma_{i}$ is chosen small enough so the frequency bandwidth of the scaled wavelets $g_{i}(t)=\frac{1}{a_{i}} g\left(\frac{t}{a_{i}}\right)$ will appreciably overlap.

A time-scale representation of data is often called a scalogram ${ }^{14}$, which is actually the power spectral density $\left|W_{g}(\tau, a)\right|^{2}$ of the signal over the $(\tau, a)$ plane. Example scalograms of a 5-20 Hz F-18 HARV aileron chirp (linear frequency sweep) input command and lateral acceleration feedback response at $50 \operatorname{deg} \alpha$ are shown in figure 2 (note $\log$ frequency scale).

Time-frequency masking of input and output is performed along the sweep. This filtering procedure on the input and output is shown in figure 3 as processed scalograms. On-board excitation system (OBES) inputs are relatively clean since they are generated digitally by the flight system, so time-frequency filtering of the output will be more significant in this case. Figure 4 shows the effect of filtering on the responses. Note that effective signal reconstruction from the processed scalograms is accomplished from the real wavelet basis.

\section{Parametric Modal Estimation - Morlet Wavelet Transform}

Modal parameters can be estimated with wavelets by analysis of the system impulse response ${ }^{18}$ (see appendix). The DWT of a signal using the complex Morlet wavelet 

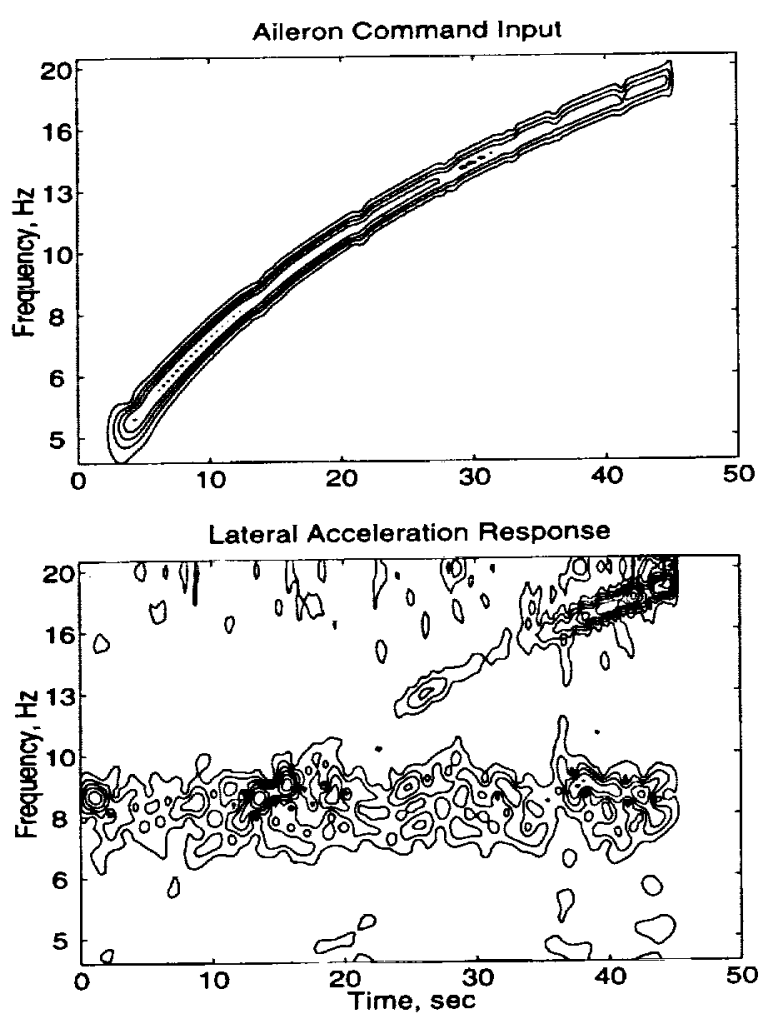

Figure 2: Scalogram contours of OBES aileron command input (top) and lateral acceleration response (bottom).
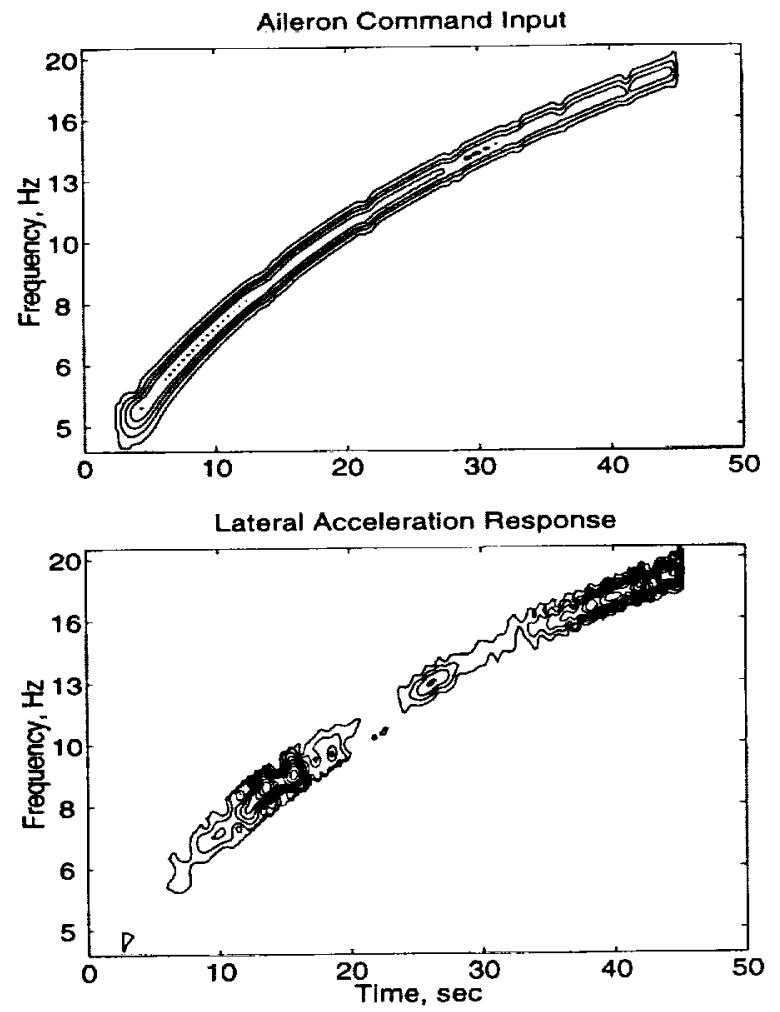

Figure 3: Scalogram contours of filtered OBES aileron command input (top) and lateral acceleration response (bottom).
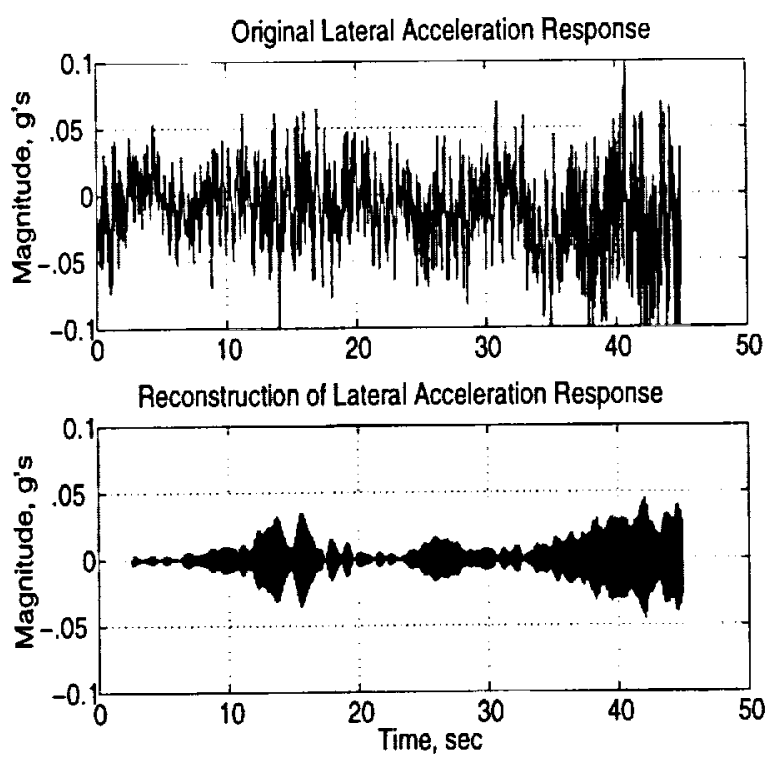

Figure 4: Responses of original (top) and filtered (bottom) lateral acceleration from OBES aileron command input.

is a complex-valued matrix whose modulus and phase are related to impulse response parameters. In the current application, this procedure is applied at every time point assuming at each instant that the response is a sum of multiple degree-of-freedom impulse responses.

An example of response frequency estimation using the linear phase variation of the WT is illustrated in figure 5 for the filtered lateral acceleration response from aileron input command at $50 \operatorname{deg} \alpha$ (data from fig. 4). The raw estimate in figure 5 (top) corresponds to the derivative of the phase variation of the WT between 20-27 sec. Hence, this is an estimate of instantaneous frequency from equation 3. Data spikes are removed by limiting values of the second derivative below some threshold. The refined estimate of figure 5 (bottom) is computed from the data of figure 5 (top) with spikes removed, and these are used to derive an approximate response frequency of $11.8 \pm 0.3 \mathrm{~Hz}$ over the respective time span. Wavelet modulus decay is used similarly to derive decay rate.

Some results of wavelet-based modal estimation are illustrated in figure 6 using the same data from the wavelet filtered results of figure 4. Figure 6 (upper left) represents the mean value of the instantaneous frequency $\phi(t)$, or estimated $\omega_{d}$, as a function of the complex Morlet wavelet frequency $\omega_{0}$. Similarly, figure 6 (upper right) plots the estimated decay rate, or frequency $\zeta \omega_{n}$, also as a function of $\omega_{0}$. From these two parameters are derived the modal natural frequency $\omega_{n}$ and modal damping ratio $\zeta$ as functions of $\omega_{0}$ in the lower left and lower right plots, respectively.

Finally, the bank of Morlet wavelets used for natural frequency and damping ratio estimation are tagged for 

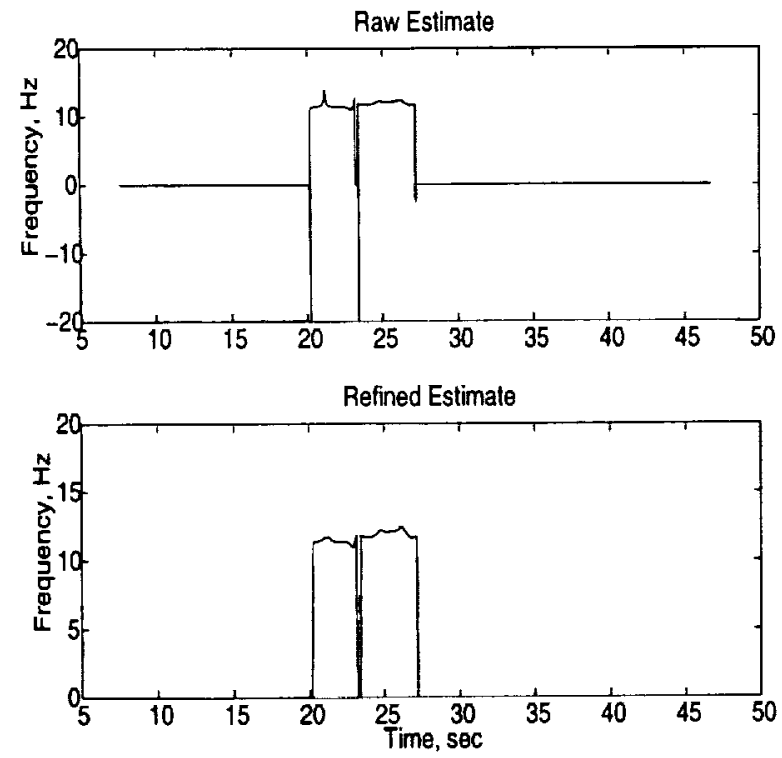

Figure 5: Instantaneous Frequency Estimation.

Raw estimate (top) and refined estimate (bottom).

starting time and duration to get the modal estimates as functions of time. Time-dependent modal parameter estimates are displayed in figure 7 . It is observed that modal frequency is essentially the tracked input frequency in this case since the cleaned output signal from figure 4 (bottom) is being used, and this response tends to track the input frequency. From the scalogram of figure 3 (bottom), the response lacks definition between 20-25 sec and 32-34 sec. These gaps also correspond to the lower output signal levels from figure 4 (bottom) at these time intervals. Lack of observability makes the modal damping results of figure 7 (right) questionable in these particular intervals.

An important point to emphasize here is that the Morlet wavelets are being used to estimate the modal parameters. Therefore, an implicit filtering process is being performed independent of the explicit procedure described previously. The wavelet basis representation of the signal is itself a noise-free subspace of the signal function space, and the modal parameters are derived from this signal subspace.

\section{$\mu$ Method}

A method to compute stability margins of aeroservoelastic systems has been formulated based on robust stability theory ${ }^{12}$. This method uses a set of structured operators $\Delta$, referred to as uncertainty, to describe errors and unmodeled dynamics in an analytical model. The structured singular value, $\mu$, is used to compute a stability margin for this model that is robust, or worst-case, to the uncertainty operators ${ }^{16}$.
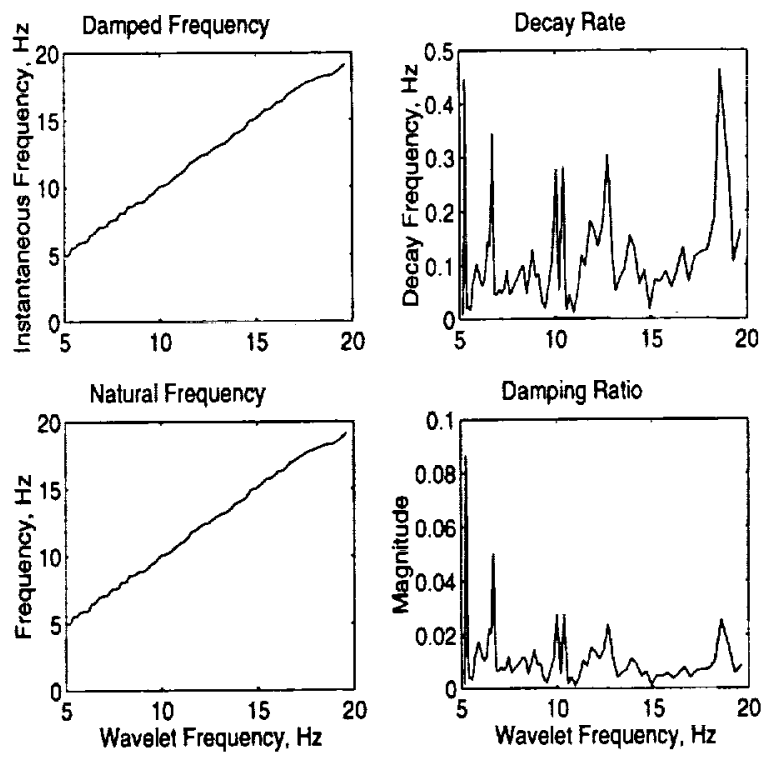

Figure 6: Modal Frequency and Damping Estimates. Damped frequency vs. wavelet frequency (upper left), decay rate vs. wavelet frequency (upper right), natural frequency vs. wavelet frequency (lower left), and damping ratio vs. wavelet frequency (lower right).
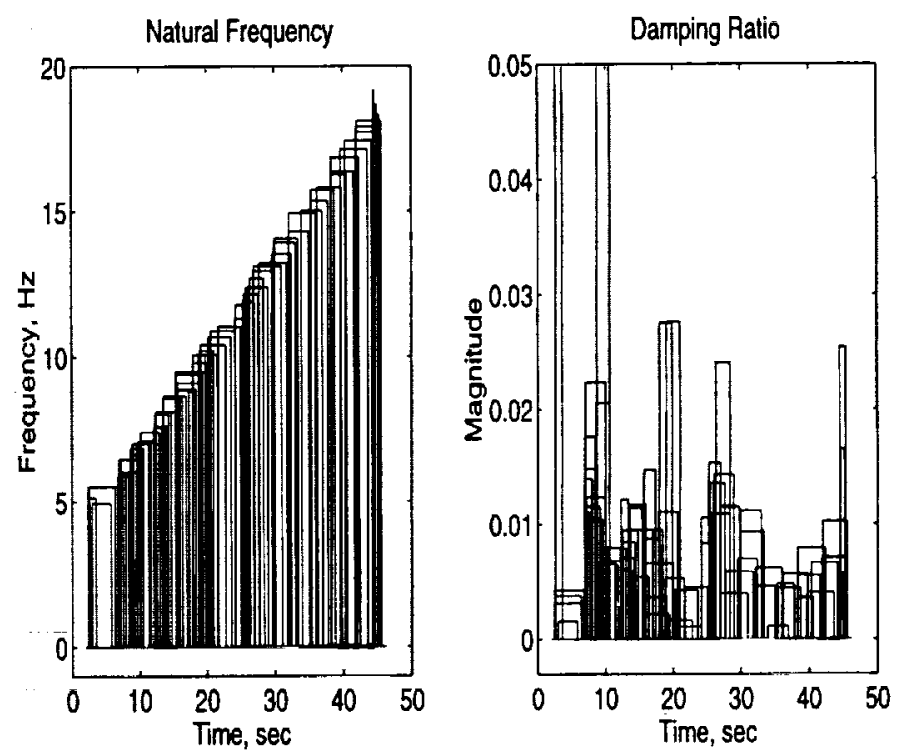

Figure 7: Response natural frequency (left) and damping ratio (right) estimates.

The $\mu$ framework represents systems as operators with interconnections known as linear fractional transformations. This paper will use the notation $F(P, \Delta)$ to represent an feedback interconnection of the plant $P$ and uncertainty $\Delta$. Aeroservoelastic systems may have errors affecting different dynamic subsystems so the uncertainty operator $\Delta$ is structured such that the feedback interconnections ensure each subsystem is affected by the proper component of $\Delta$. 
Flight data can be incorporated into the $\mu$ method by formulating an uncertainty description that accounts for observed variations and errors ${ }^{11}$. A model validation analysis is performed on the plant model to ensure the range of dynamics admitted by the uncertainty is suffcient to cover the range of dynamics observed with the flight data. Thus, a robust stability margin is computed that directly accounts for flight data.

A flowchart for information processing by the $\mu$ method is shown in figure 8 .

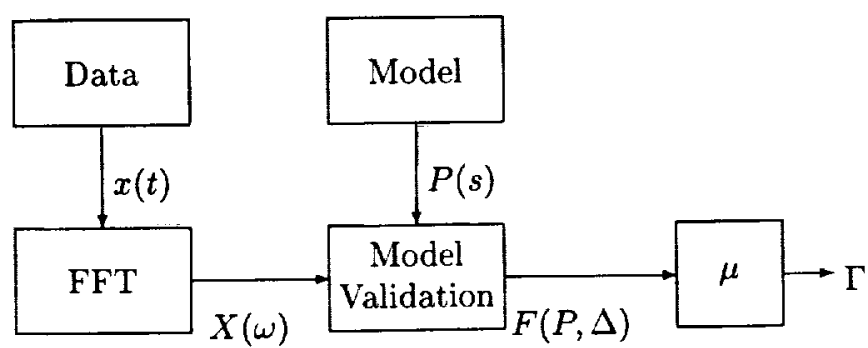

Figure 8: Flowchart of $\mu$ method for robust stability margin analysis of ASE dynamics.

An ASE stability margin, $\Gamma$, is determined by computing $\mu$ with respect to an uncertainty description, $\delta_{\bar{q}}$, that admits variations in dynamic pressure $(\bar{q})$ and an uncertainty description, $\Delta$, that describes modeling errors ${ }^{10}$. This margin relates the largest change in dynamic pressure that may be considered while guaranteeing the plant model is robustly stable to all errors described by $\Delta$.

\section{$\mu$ Method with Wavelet Processing}

The $\mu$ method can be coupled with the wavelet filtering processes of parametric and nonparametric estimation discussed previously. This coupling is achieved by introducing several time-frequency operations based on wavelet filtering into the basic process. The general information flowchart for the $\mu$ method with wavelet filtering is shown in figure 9 .

Wavelet transform operations are introduced to process the time domain data, $x(t)$, before a frequency domain representation, $\hat{X}(\omega)$, is computed. These operations map the time domain data into a time-frequency domain scalogram via a wavelet transform and then map a scalogram back into the time domain via an inverse wavelet transform. A time-frequency filtering process operates between the WT and inverse WT to remove unwanted features from the scalogram before the inverse WT computes a time domain signal, $\hat{x}(t)$.

A modal parameter estimation operation is introduced using the wavelet algorithm. Properties of the system dynamics are derived from the filtered scalogram. The

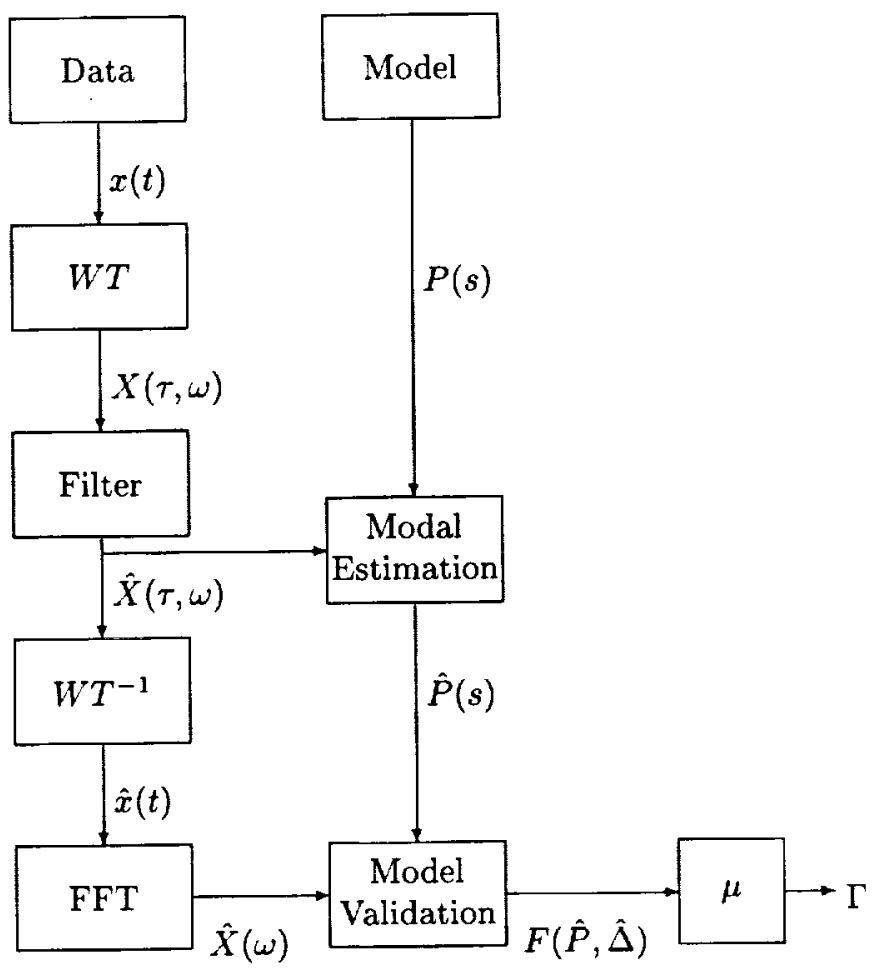

Figure 9: Flowchart of $\mu$ method with wavelet filtering for robust stability margin analysis of ASE dynamics.

elements of a nominal plant model, $P$, are updated with these parameter estimates and a new plant model, $\hat{P}$, is used to represent the aeroservoelastic dynamics.

The final operations of the $\mu$ method are traditional robust stability operations that operate on frequency domain data. The effect of the wavelet filtering is to use the filtered versions of the data and plant model for the modal validation. Thus, a new uncertainty operator, $\hat{\Delta}$, is associated with the parameter updated plant, $\hat{P}$, to account for errors observed from the filtered data, $\hat{x}(t)$. A robust stability margin, $\Gamma$, is computed that describes the largest change in dynamic pressure for which $\hat{P}$ is robustly stable to the errors $\hat{\Delta}$.

\section{$\mu$ Method with Parameter Estimation}

An implementation of the $\mu$ method with modal parameter estimation is accomplished using the flowchart of figure 9. The filter operation for this implementation is ignored so the wavelet map $\hat{X}(\tau, \omega)$ is equivalent to the original map $X(\tau, \omega)$.

The wavelet based method for parametric estimation is used to analyze the wavelet map $\hat{X}(\tau, \omega)$ of the flight data. This method estimates modal parameters to describe the system dynamics that generated the flight data. A plant model, $\hat{P}_{1}$, is computed by updating elements of the nominal plant model, $P_{0}$, with the modal parameter estimates. Only a limited subset of dynamics 
will be observable in the data so only a correspondingly limited subset of the plant modal parameters will be updated.

An uncertainty description, $\hat{\Delta}_{1}$, is generated for the plant with updated modal parameters, $\hat{P}_{1}$, using the model validation procedure. This procedure essentially uses the original flight data measurements since the WT and inverse WT operations will cancel each other except for numerical inaccuracies. Thus, $x(t) \approx \hat{x}(t)$ and an uncertainty description is computed for the updated plant which accounts for all variations and anamolies in the recorded data.

The magnitude of uncertainty associated with the updated plant should be less than (or equal to) the uncertainty magnitude associated with the nominal plant. This decrease in uncertainty results from the ability of the updated plant to account for bias in the nominal plant estimates. Consider the uncertainty balls needed to cover parameter values and measurements shown in figure 10.
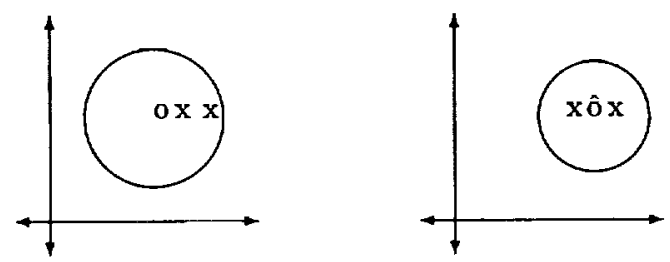

Figure 10: Balls of uncertainty covering nominal value (o) and measurements $(x)$ (left) and parameter estimation value $(\hat{o})$ and measurements $(x)$ (right).

The left plot of figure 10 shows the uncertainty ball associated with a nominal parameter, indicated by $(o)$, to account for the measured parameters, indicated by $(x)$. The right plot shows the uncertainty ball associated with an updated parameter estimate, indicated by $(\hat{o})$, to account for the same measured parameters. Clearly the uncertainty associated with the updated parameter is less than the uncertainty associated with the nominal parameter. Thus, $\left\|\hat{\Delta}_{1}\right\|_{\infty} \leq\left\|\Delta_{0}\right\|_{\infty}$.

The conservatism in robust stability margins computed by the $\mu$ method arises from the excessive uncertainty needed to account for errors in a model. A decrease in uncertainty from model updating with the parameter estimation process should decrease this conservatism.

\section{$\mu$ Method with Wavelet Filtering and \\ Parameter Estimation}

Another implementation of the $\mu$ method with modal parameter estimation results from including a nontrivial filtering operation in the flowchart of figure 9. The wavelet filtering operation, which is a type of nonparametric estimation, is used to generate scalograms to rep- resent desired features of input and output data in the time-frequency domain. The filtered scalogram, $\hat{X}(\tau, \omega)$, may be arbitrarily different than the original scalogram, $X(\tau, \omega)$, depending on the energy of the signal components which do not correlate to desired features.

The filtered wavelet map is input to the parametric estimation process. Resulting modal parameter estimates represent the dynamics of the system model that generates the desired features dominant in the filtered maps. The elements of the nominal plant model, $P_{0}$, are replaced with the modal parameter estimates to generate an updated plant model, $\hat{P}_{2}$.

The filtered wavelet map is also used to generate an uncertainty description for the updated plant $\hat{P}_{2}$. A time domain signal, $\hat{x}(t)$, which represents the filtered measurement data is computed by an inverse wavelet transform on the filtered scalogram. A frequency domain representation of this filtered signal is computed from a Fourier transform and is used by the model validation process. The resulting uncertainty, $\hat{\Delta}_{2}$, describes the variations between the updated plant $\hat{P}_{2}$ and the filtered data.

The uncertainty description associated with $\hat{P}_{2}$ should be less (or equal) when validating the filtered data as compared to validating the unfiltered data. The filtering process should remove nonlinearities and harmonics along with noise that causes aliasing and errors in measured transfer functions. This removal of errors may decrease the variance in modal parameter estimates so that an updated model can be generated with less uncertainty. Consider the example balls of uncertainty associated with a parameter shown in figure 11 .
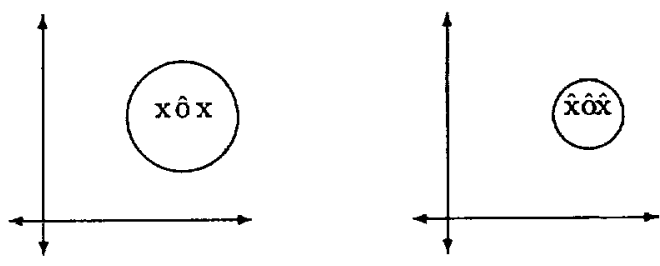

Figure 11: Balls of uncertainty covering parameter estimation value $(\hat{o})$ and measurements $(x)$ (left) and parameter estimation value $(\hat{o})$ and filtered measurements $(\hat{x})$ (right).

The left plot of figure 11 shows the uncertainty ball associated with a parameter, indicated by $(\hat{o})$, to account for the measured parameters, indicated by $(x)$. The right plots shows the uncertainty ball associated with the same parameter but accounting for parameters obtained from filtered data, indicated by $(\hat{x})$. The filtered data generate parameters that are less scattered allowing the uncertainty ball to be smaller. The filtering process may actually cause greater scattering; however, it is assumed data measurements with the least scatter will be used for model validation so $\left\|\hat{\Delta}_{2}\right\|_{\infty} \leq\left\|\hat{\Delta}_{1}\right\|_{\infty} \leq\left\|\Delta_{0}\right\|_{\infty}$. 
The conservatism in robust stability margins computed by the $\mu$ method may be decreased by including the wavelet filtering into the process.

\section{Aircraft Models and Uncertainties}

Robust stability margins for the aeroservoelastic dynamics of the F-18 HARV are computed using the $\mu$ method with wavelet filtering. Stability margins are computed for the antisymmetric modes (table 1) of the lateraldirection aeroservoelastic dynamics for the aircraft at Mach 0.3 and an altitude of $30,000 \mathrm{ft}\left(\bar{q}=41 \mathrm{lb} / \mathrm{ft} \mathrm{t}^{2}\right)$ at $50 \operatorname{deg} \alpha$. A baseline implementation of the $\mu$ method indicates these margins may lie within the flight envelope so any reduction in conservatism could be significant at this flight condition ${ }^{12}$.

An uncertainty description is formulated using three operators to describe errors in an F-18 HARV analytical model. A complex operator, $\Delta_{i n}$, is a multiplicative uncertainty in the control inputs to the plant and accounts for actuator errors and unmodeled dynamics. Another complex operator, $\Delta_{a d d}$, relates the control inputs to the feedback measurements to account for uncertainty in the magnitude and phase of the computed plant responses. The remaining uncertainty operator, $\Delta_{A}$, is a real parametric uncertainty affecting the modal parameters of the open-loop state matrix to describe errors in natural frequency and damping parameters.

The block diagram for robust stability analysis of the F-18 HARV aeroservoelastic dynamics is shown in figure 12. This figure includes an operator, $\delta_{\vec{q}}$, that affects the nominal dynamics to describe changes in flight condition and is used to interpret $\mu$ as a stability margin ${ }^{10}$. Additional operators, $W_{a d d}$ and $W_{i n}$, are shown as weightings to normalize the frequency varying uncertainty operators, $\Delta_{a d d}$ and $\Delta_{i n}$. The system model also contains $2 \%$ sensor noise corruption on each measurement.

The lateral-directional controller, $K$, has 29 states. The feedback measurements and control inputs associated with this controller are given in table 2 .

\begin{tabular}{l|l}
\hline \hline roll rate & aileron \\
yaw rate & differential leading edge flap \\
dideslip rate & diffential trailing edge flap \\
lateral acceleration & $\begin{array}{l}\text { rudder } \\
\text { yaw thrust vectoring }\end{array}$ \\
\hline
\end{tabular}

Table 2: Feedback measurements (left) and control commands (right) for the thrust vectoring lateral-directional controller $K$.

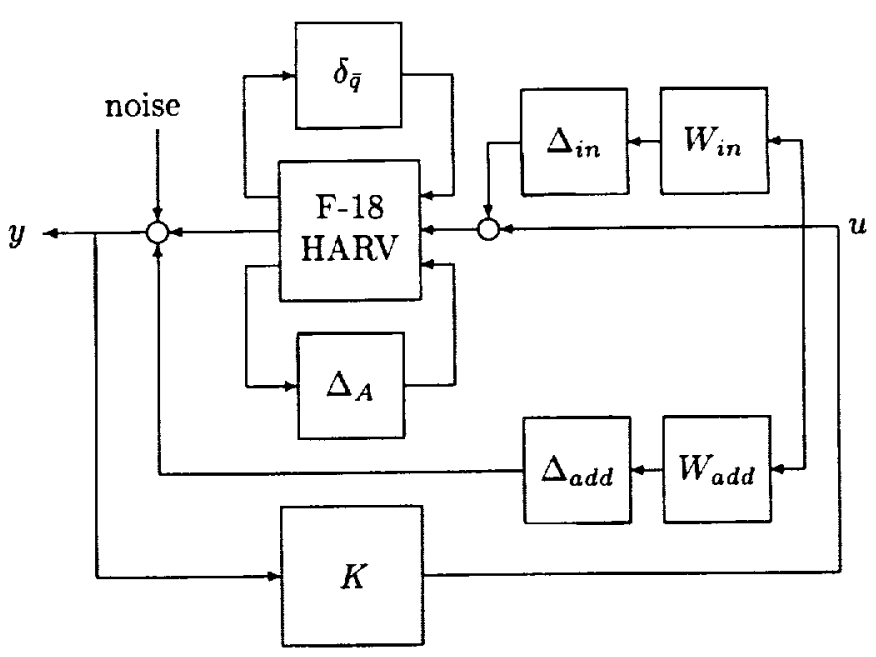

Figure 12: F-18 HARV uncertainty block diagram for robust stability margin analysis.

\section{Baseline Model Validation}

A model with an associated uncertainty description was generated to compute robust stability margins by the $\mu$ method. The plant model, $P_{0}$, is the nominal model generated by a finite element analysis of the aeroservoelastic dynamics. The parameters in this model are theoretical and have not been updated by analysis of flight data. The model contains seven antisymmetric elastic structural modes between 5 and $20 \mathrm{~Hz}$ (table 1).

An uncertainty description, $\Delta_{0}$, is generated using the model validation procedure on a frequency domain representation of the unfiltered data as in figure 8 . Only the observed energies from frequencies below $20 \mathrm{~Hz}$ are used for validation because there is considerable energy at frequencies near $20 \mathrm{~Hz}$ caused by structural dynamics associated with the thrust-vectoring vane system that are difficult to model. The primary transfer function used in the derivation of the uncertainty description is the lateral acceleration response from yaw thrust vectoring. These data responses demonstrate good observability of the primary modes up to $20 \mathrm{~Hz}$.

Separate parametric uncertainty levels are chosen for each mode of the open-loop state matrix to reflect different levels of accuracy. These uncertainty magnitudes are computed to describe observed variations between the model transfer function and the flight data measurements. The nominal modal parameters and the amount of variation admitted by the parametric uncertainty are given in table 3 .

The amount of variation needed to describe modal parameter errors is fairly significant for all modes, especially in damping ratio. The fuselage first torsion and 


\begin{tabular}{crc}
\hline \hline Mode & $\omega(\mathrm{Hz})$ & $\zeta$ \\
\hline Fuselage $1^{\text {st }}$ Bending & $6.85 . \pm .07$ & $.012 \pm .006$ \\
Wing $1^{\text {st }}$ Bending & $8.96 \pm .18$ & $.006 \pm .004$ \\
Wing 1 $^{\text {st }}$ Torsion & $12.84 \pm .13$ & $.011 \pm .006$ \\
Wing Fore-Aft & $15.69 \pm .63$ & $.010 \pm .007$ \\
Fuselage ${ }^{\text {st }}$ Torsion & $18.86 \pm .76$ & $.010 \pm .005$ \\
\hline \hline
\end{tabular}

Table 3: Modal parameters and uncertainty variations for model $P_{0}$ and $\Delta_{0}$.

wing fore-aft modes have properties which are particularly poorly modeled so there is up to $4 \%$ error in natural frequency and $70 \%$ error in damping. The remaining modes have only $2 \%$ error in natural frequency but still require at least $50 \%$ error in damping.

The weighting functions for the input multiplicative and additive uncertainties are chosen to account for any errors between the model and the flight data that can not be covered by the parametric modal uncertainty.

$$
\begin{gathered}
W_{\text {in }}=10 \frac{s+100}{s+5000} \\
W_{\text {add }}=.02
\end{gathered}
$$

\section{Model Validation with Parameter Estimation}

The parametric modal estimation procedure was used to process the flight data and compute modal parameters for an analytical model. This procedure uses equation 2 to generate estimates of the modal parameters from the unfiltered wavelet map $X(\tau, \omega)$ and associated properties.

A plant model, $P_{1}$, is computed which is the estimated plant model obtained from the wavelet filtering. This model is formulated initially as the nominal plant $P_{0}$ but with certain theoretical modal parameters replaced by their estimated values. The nominal values of these parameters are shown in table 4 . The natural frequencies are not changed by more than $1 \mathrm{~Hz}$ for any of the estimated modes; however, the estimated damping parameters are significantly higher than the theoretical values.

An uncertainty description, $\Delta_{1}$, is associated with $P_{1}$ to describe the levels of modeling error in this estimated plant. The magnitudes of the parametric modal uncertainty in $\Delta_{1}$ are chosen by comparing flight data with theoretical transfer functions for $P_{1}$. The ranges of modal parameter variations admitted by this uncertainty are given in table 4 .

The variations in both natural frequency and dampings are seen to be considerably reduced in table 4 for $F\left(P_{1}, \Delta_{1}\right)$ as compared to the large variations in table 3 for $F\left(P_{0}, \Delta_{0}\right)$. The estimated modal parameters used

\begin{tabular}{crc}
\hline \hline Mode & $\omega(\mathrm{Hz})$ & $\zeta$ \\
\hline Fuselage $1^{\text {st }}$ Bending & $6.85 \pm .07$ & $.012 \pm .006$ \\
Wing $1^{\text {st }}$ Bending & $8.60 \pm .10$ & $.040 \pm .021$ \\
Wing $1^{\text {st }}$ Torsion & $13.31 \pm .15$ & $.045 \pm .024$ \\
Wing Fore-Aft & $16.51 \pm .35$ & $.045 \pm .023$ \\
Fuselage $1^{\text {st }}$ Torsion & $18.21 \pm .37$ & $.030 \pm .010$ \\
\hline \hline
\end{tabular}

Table 4: Modal parameters and uncertainty variations for model $P_{1}$ and $\Delta_{1}$.

in $P_{1}$ are much closer to those of the aircraft so the predicted response of $P_{1}$ closely matches the flight data measurements. Thus, the natural frequency errors are all less than $2 \%$ while the damping errors are all less than $55 \%$.

The weightings, $W_{a d d}$ and $W_{i n}$, affecting the remaining uncertainties in $\Delta_{1}$ are identical to those of $\Delta_{0}$.

\section{Model Validation with Wavelet Filtering and Parameter Estimation}

Modal parameters for model estimate $\hat{P}_{2}$ are extracted from the time-frequency domain representation of the wavelet-filtered flight data $\hat{X}(\tau, \omega)$. This is the procedure outlined in figure 9 . As seen in table 5 , the modal estimates from the filtered data are similar to the unfiltered estimates of table 4. Parameter variations, however, resulting from validated model $F\left(\hat{P}_{2}, \hat{\Delta}_{2}\right)$ are reduced in modal frequency to $1 \%$ and in modal damping to $10 \%$.

\begin{tabular}{crc}
\hline \hline \multicolumn{1}{c}{ Mode } & $\omega(\mathrm{Hz})$ & $\zeta$ \\
\hline Fuselage $1^{\text {st }}$ Bending & $6.85 \pm .07$ & $.012 \pm .001$ \\
Wing 1 $1^{\text {st }}$ Bending & $8.70 \pm .09$ & $.035 \pm .003$ \\
Wing $1^{\text {st }}$ Torsion & $13.31 \pm .14$ & $.045 \pm .004$ \\
Wing Fore-Aft & $16.61 \pm .17$ & $.045 \pm .004$ \\
Fuselage $1^{\text {st }}$ Torsion & $18.21 \pm .18$ & $.040 \pm .004$ \\
\hline
\end{tabular}

Table 5: Modal parameters and uncertainty variations for model $P_{2}$ and $\Delta_{2}$.

\section{ASE Stability Margins}

Nominal stability margins are computed for the plant model using the original theoretical modal parameters and the updated models using parameters estimated from wavelet filtering. These margins are computed from a $\mu$ analysis with respect to the variation in dynamic pressure, $\bar{q}$, but ignoring the modal and complex uncertainty operators. The nominal stability margins, $\Gamma$, are given in table 6 and demonstrate the largest decrease relative to the nominal dynamic pressure of $\bar{q}=41 \mathrm{lb} / \mathrm{ft}^{2}$ that may be considered before the models incur an ASE instability. Therefore, a larger negative margin indicates a greater margin of robust stability.

The original theoretical model has a nominal stability 


\begin{tabular}{ccc}
\hline \hline Model & $\Gamma$ & $\omega$ \\
\hline$F\left(P_{0}, 0\right)$ & $-268 \mathrm{lb} / \mathrm{ft}^{2}$ & $14.8 \mathrm{~Hz}$ \\
$F\left(P_{1}, 0\right)$ & $-368 \mathrm{lb} / \mathrm{ft}^{2}$ & $14.8 \mathrm{~Hz}$ \\
$F\left(P_{2}, 0\right)$ & $-379 \mathrm{lb} / \mathrm{ft}^{2}$ & $14.8 \mathrm{~Hz}$ \\
\hline \hline
\end{tabular}

Table 6: Nominal stability margins for models.

margin of $\Gamma=-268 \mathrm{lb} / \mathrm{ft}^{2}$ resulting from a critical instability of the wing fore-aft mode at $14.8 \mathrm{~Hz}$. The margins are increased by updating the models with modal parameters estimates; however the wing fore-aft mode remains the critical mode for these updated models. This increase in stability margin associated with wavelet filtering is not guaranteed to occur for all applications; rather, the filtering is designed to make the nominal model more accurate. The nominal model for the F-18 HARV has excessively low damping values compared to the damping levels resulting from the wavelet filtering. Increasing damping ratio estimates make the plant effectively more stable and increase the stability margins.

These nominal margins are all greater in absolute value than the nominal dynamic pressure so they demonstrate the nearest instability to the flight envelope occurs at a negative dynamic pressure, which is physically unrealizable. Thus, the nominal dynamics are free of ASE instabilities within the research flight envelope.

Robust stability margins are computed with respect to the uncertainty description of figure 12 and given in table 7 . Model $F\left(P_{0}, \Delta_{0}\right)$ describes the original model with parameter variations as in table 3 . The model with modal parameter estimates, $F\left(P_{1}, \Delta_{1}\right)$, has the reduced uncertainty levels leading to the variations in table 4 . The remaining model, $F\left(P_{2}, \Delta_{2}\right)$, describes the model formulated by combining wavelet filtering with parameter estimation and introducing uncertainty to allow the variations in table 5.

\begin{tabular}{ccc}
\hline \hline Model & $\Gamma$ & $\omega$ \\
\hline$F\left(P_{0}, \Delta_{0}\right)$ & $-4 l b / f t^{2}$ & $15.4 \mathrm{~Hz}$ \\
$F\left(P_{1}, \Delta_{1}\right)$ & $-222 \mathrm{lb} / \mathrm{ft}^{2}$ & $7.0 \mathrm{~Hz}$ \\
$F\left(P_{2}, \Delta_{2}\right)$ & $-239 \mathrm{lb} / \mathrm{ft}^{2}$ & $7.0 \mathrm{~Hz}$ \\
\hline \hline
\end{tabular}

Table 7: Robust stability margins for models with respect to uncertainty descriptions.

The stability margin of the original model is strongly affected by considering uncertainty. This margin is reduced from $\Gamma=-268 \mathrm{lb} / \mathrm{ft}^{2}$ for the nominal dynamics to $\Gamma=-4 l b / f t^{2}$ for the dynamics with respect to uncertainty. The critical mode remains the wing fore-aft mode despite the uncertainty; however, the dynamic pressure at which this mode becomes unstable is quite different. This robust stability margin demonstrates the nominal model may be misleading and the nearest unstable flight condition may actually lie within the flight envelope.

The robust stability margin for the model $F\left(P_{1}, \Delta_{1}\right)$, using modal parameter estimates, is significantly larger than the margin of the original system. The wavelet processing is able to identify a more accurate model with less associated uncertainty so the conservatism in the margin is reduced. The robust stability margin for this model is $\Gamma=-222 \mathrm{lb} / \mathrm{ft}^{2}$ and indicates the nearest instability for the updated model. Despite the range of dynamics incurred by uncertainty, the margin is at a negative dynamic pressure and so the flight envelope is free of ASE instabilities.

The critical mode associated with the robust stability margin for the updated model is the first fuselage bending mode. This differs from the critical wing fore-aft mode associated with the nominal margin. This shift in critical mode is a result of modal parameter updates and corresponding reduced uncertainty sets.

The model formulated from parameter estimation coupled with wavelet filtering, $F\left(P_{2}, \Delta_{2}\right)$, has a robust stability margin which is similar to the margin of $F\left(P_{1}, \Delta_{1}\right)$. The magnitude of this margin is slightly higher as a result of the reduced uncertainty levels needed to validate the filtered flight data; however, the critical mode remains the fuselage bending mode.

Reduction in parameter variations from nonparametric wavelet filtering did not have as much an effect on robust stability as the updated parameter estimates. Nonparametric filtering has more impact on parameter variance which was a less significant factor than parameter bias.

To summarize, comparison between the nominal results in table 6 and the robust margins of table 7, shows that the decrease in margin from uncertainty is clearly evident. The decrease is most substantial for plant model $P_{0}$, which has the greatest amount of modal uncertainty in $\Delta_{0}$, yet the frequency of instability is consistent with the nominal cases. When updated modal parameter estimates are incorporated in $P_{1}$ and $P_{2}$, the decrease in margins compared to the nominal models are somewhat less because of the smaller uncertainty sets $\left(\Delta_{1}, \Delta_{2}\right)$ compared to $\Delta_{0}$.

The main difference between nominal and robust results is in modal frequency of instability. Wing fore-aft modal frequency increased about $1 \mathrm{~Hz}$ from its theoretical value to the updated value, and thereby became a less significant factor in the stability margin calculation compared with first fuselage bending. This result confirms that the effect of parameter estimation, and essentially data quality, in model validation becomes a critical factor in robust stability boundary prediction. 


\section{Conclusions}

Improvements in aeroservoelastic flight data analysis and stability prediction estimation have been addressed. Wavelet approaches to system identification were applied by combining both filtering and parametric timefrequency identification algorithms with Morlet wavelets. The combination of these estimation schemes extracted modal estimates and system uncertainty representations for less conservative model validation. Uncertainty ranges validated by F-18 HARV ASE data were shown to decrease by incorporating modal estimates based on the wavelet-processed data.

With the model parameter and uncertainty description updates, the critical aeroservoelastic instability changed in modal frequency and flight condition. A predicted instability within the flight envelope using an uncertain baseline model was found to be too conservative. Model updates pushed the instability much beyond the flight regime. The ultimate objective of predicting stability boundaries from flight data was enhanced by a reduction in conservatism of the stability margin estimates.

\section{Appendix}

Given a general harmonic signal

$$
x(t)=k(t) \cos (\phi(t) t)
$$

the WT of $x(t)$ is

$$
W(a, \tau)=\sqrt{a} k(t) e^{-\left(a \phi(t)-\omega_{0}\right)^{2}} e^{i \phi(t) \tau} .
$$

For fixed dilation parameter $a_{i}$ (equivalently fixed frequency $\omega)$, the modulus and phase of the WT of $x(t)$ are

$$
\begin{aligned}
\left|W\left(a_{i}, \tau\right)\right| & =\sqrt{a_{i}} k(t) e^{-\left(a_{i} \phi(t)-\omega_{0}\right)^{2}} \\
\left.L W\left(a_{i}, \tau\right)\right] & =\phi(t) \tau
\end{aligned}
$$

Instantaneous frequency of a signal in this case can be expressed as ${ }^{18}$

$$
\phi(t)=\frac{1}{2 \pi} \frac{d}{d t}\left(\angle\left[W\left(a_{i}, \tau\right)\right]\right)
$$

This shows that a general time-varying envelope $k(t)$ or phase $\phi(t)$ of the signal can be determined from the modulus and phase of the WT for each fixed wavelet frequency.

More specifically, from the impulse response of a single degree-of-freedom viscous damper

$$
x(t)=A e^{-\zeta \omega_{n} t} \cos \left(\omega_{d} t+\phi_{o}\right)
$$

we have from equation 2 ,

$$
\begin{aligned}
& k(t)=\frac{\left|W\left(a_{i}, \tau\right)\right|}{\sqrt{a_{i} e^{-\left(a_{i} \phi(t)-\omega_{0}\right)^{2}}}}=A e^{-\zeta \omega_{n} t} \\
& \phi(t) t=\angle\left[W\left(a_{i}, \tau\right)\right]=\omega_{d} t+\phi_{o}
\end{aligned}
$$

For a constant wavelet frequency line corresponding to $a_{i}$ over time $\tau$ in the $(a, \tau)$ plane, estimation of the WT linear phase variation (or mean value of the instantaneous frequency over time, from eq. 3) gives $\phi(t) \approx \omega_{d}$, and the envelope decay rate is $\zeta \omega_{n}$. Natural frequency $\omega_{n}$ and modal damping ratio $\zeta$ are therefore derived. The WT becomes a complex representation of the original real signal from which the signal eigenvalues are computed without any approximation of their range.

Multiple degree-of-freedom systems are analyzed similarly by noting that the dilated Morlet wavelet is a bandpass filter (eq. 1). With sufficient resolution of dilation $a_{i}$, damped modal frequencies $\omega_{d_{i}}=\frac{\omega_{0}}{a_{i}}$ can be discriminated. To recap, the decay rate of the envelope of each mode is calculated from the log-slope of the wavelet modulus decay, and damped modal frequency is estimated as the linear phase variation of the WT as a function of time. Adequate frequency resolution can be enforced with the multiscaled compact harmonic Morlet wavelets.

\section{References}

1. Bowers, Albion H., Joseph W. Pahle, Joseph R. Wilson, Bradley C. Flick, and Richard L. Rood, $A n$ Overview of the NASA F-18 High Alpha Research Vehicle, NASA-TM-4772, Oct. 1996.

2. Brenner, Martin J. and Eric Feron, "Wavelet Analysis of F/A-18 Aeroelastic and Aeroservoelastic Flight Test Data," AIAA Structures, Structural Dynamics, and Materials Conference, AIAA 971216 and NASA TM-4793, Apr 1997.

3. Brenner, Martin J., Richard C. Lind, and David F. Voracek, "Overview of Recent Flight Flutter Testing Research at NASA Dryden," AIAA Structures, Structural Dynamics, and Materials Conference, AIAA 97-1023 and NASA TM-4792, Apr 1997.

4. Brenner, Martin J., Aeroservoelastic Modeling and Validation of a Thrust-Vectoring F/A-18 Aircraft, NASA TP-3647, Sep 1996.

5. Feron, Eric, Marty Brenner, Jim Paduano, and Arkediy Turevskiy, "Time-frequency Analysis for Transfer Function Estimation and Application to the F18-SRA," accepted to AIAA Journal of Guidance, Control, and Dynamics, 21(2), Mar-Apr 1998. 
6. Freudinger, Lawrence C., Rick Lind, and Martin J. Brenner, "Correlation Filtering of Modal Dynamics using the Laplace Wavelet," International Modal Analysis Conference, Feb 1998.

7. Le, Dzu K., "Application of Sampling Theorems in Wavelet Spaces to Multiresolution Visualization and Data Segmentation," SPIE Proceedings of Wavelet Applications in Signal and Image Processing III, Vol. 2569, Jul 1995, pp. 220-233.

8. Le, Dzu K., Albert K. Owen, and Dyane L. Mattern, "Multiscale Analysis of Stall Inception and Instabilities in an Axi-Centrifugal Turboshaft Engine," AIAA Joint Propulsion Conference, AIAA 96-3174, Jul 1996.

9. Lind, Rick, Marty Brenner, and Sydney Haley, "Estimation of Modal Parameters using a Waveletbased Approach," AIAA Atmospheric Flight Mechanics Conference, AIAA 97-3836, Aug 1997.

10. Lind, Rick and Marty Brenner, "Robust Flutter Margins of an F/A-18 Aircraft from Aeroelastic Flight Data," AIAA Journal of Guidance, Control, and Dynamics, 20(3), May-Jun 1997, pp. 597-604.

11. Lind, Rick and Marty Brenner, "Incorporating Flight Data into a Robust Aeroelastic Model," accepted to AIAA Journal of Aircraft, 35(2), MarApr 1998.

12. Lind, Rick and Marty Brenner, "Analysis of Aeroservoelastic Stability Margins using the $\mu$ Method," AIAA Structures, Structural Dynamics, and Materials Conference, AIAA-98-1895, Apr 1998.

13. Mallat, Stéphane and Zhifeng Zhang, "Matching Pursuit with Time-Frequency Dictionaries," IEEE Transactions on Signal Processing, 41(12), Dec 1993, pp. 3397-3415.

14. Meyer, Yves, Wavelets: Algorithms and Applications, translated by Robert D. Ryan, SIAM, 1993.

15. Newland, David, An Introduction to Random Vibrations, Spectral and Wavelet Analysis, 3rd edition, Addison Wesley Longman, 1993.

16. Packard, Andrew K. and John C. Doyle, "The Complex Structured Singular Value," Automatica, 29(1), 1993, pp. 71-109.

17. Pahle, Joseph W., Bruce Powers, Victoria Regenie, Vince Chacon, Steve Degroote and Steven Murnyack, Research Flight Control Development for the F-18 High-Alpha Research Vehicle, NASA TM-104232, 1991.
18. Ruzzene, M., A. Fasana, L. Garibaldi, and B. Piombo, "Natural Frequencies and Dampings Identification using Wavelet Transform: Application to Real Data," Mechanical Systems and Signal Processing (1997), 11(2), pp. 207-218.

19. Shensa, Mark J., "The Discrete Wavelet Transform: Wedding the $\grave{A}$ Trous and Mallat Algorithms," IEEE Transactions on Signal Processing, 40(10), Oct 1992, pp. 2464-2482.

20. Shensa, Mark J., "Discrete Inverses for Nonorthogonal Wavelet Transforms," IEEE Transactions on Signal Processing, 44(4), Apr 1996, pp. 798-807.

21. Xia, Xiang-Gen, "System Identification Using Chirp Signals and Time-Variant Filters in the Joint Time-Frequency Domain," IEEE Transactions on Signal Processing, 45(8), Aug 1997, pp. 2072-2084. 

Public reporting burden for this collection of information ls estimated to average 1 hour per response, Including the time for reviewing instructions, searching existing data sources, gathering and maintaining the data needed, and completing and reviewing the collection of information. Send comments regarding this burden estimate or any olther aspect of this collection of information, including suggestions bor reducing this burden, to Washington Headquerters Services, Directorate for Information Operations and Reports, 1215 Jefferson Davis Highway, Suite 1204, Arlington,
VA $22202-4302$, and to the Otice of Managerment and Budget, Paperwork Reduction Project (0704-0186), Washington, DC 20503.

\begin{tabular}{|l|l|l|}
\hline 1. AGENCY USE ONLY (Loave blank) & $\begin{array}{l}\text { 2. REPOHT DATE } \\
\text { March } 1998\end{array}$ & $\begin{array}{l}\text { 3. REPORT TYPE AND DATES COVEAED } \\
\text { Technical Memorandum }\end{array}$ \\
\hline
\end{tabular}

\begin{tabular}{|l|l}
\hline 4. TIILE AND SUBTILE & 5. FUNDING NUMBERS
\end{tabular}

Wavelet Filtering to Reduce Conservatism in Aeroservoelastic Robust

Stability Margins

6. AUTHOR(S)

WU 5295004

Marty Brenner and Rick Lind

7. PERFOAMING ORGANIZATION NAME(S) AND ADDRESS(ES)

8. PERFOAMING ORGANIZATION

REPORT NUMBER

NASA Dryden Flight Research Center

P.O. Box 273

$\mathrm{H}-2222$

Edwards, California 93523-0273

10. SPONSORINGMONITORING

9. SPONSOAINGMONTTORING AGENCY NAME(S) AND ADDRESS(ES)

AGENCY REPORT NUMBER

National Aeronautics and Space Administration

Washington, DC 20546-0001

NASA/TM-1998-206545

11. SUPPLEMENTARY NOTES

Rick Lind is a National Research Council research associate. AIAA 98-1896 - presented at the AIAA

Structures, Structural Dynamics and Materials Conference, Long Beach, Califomia, April 20-23, 1998.

12a. DISTRIBUTIONAVAILABILTY STATEMENT

12b. DISTRIBUTION CODE

Unclassified-Unlimited

Subject Category 08

13. ABSTRACT (Maximum 200 words)

Wavelet analysis for filtering and system identification was used to improve the estimation of aeroservoelastic stability margins. The conservatism of the robust stability margins was reduced with parametric and nonparametric time-frequency analysis of flight data in the model validation process. Nonparametric wavelet processing of data was used to reduce the effects of external desirableness and unmodeled dynamics. Parametric estimates of modal stability were also extracted using the wavelet transform. Computation of robust stability margins for stability boundary prediction depends on uncertainty descriptions derived from the data for model validation. F-18 High Alpha Research Vehicle aeroservoelastic flight test data demonstrated improved robust stability prediction by extension of the stability boundary beyond the flight regime.

\section{SUBJECT TEAMS}

Aeroservoelasticity, Modal estimation, Robust stability, Uncertainty modeling, Wavelet analysis

\begin{tabular}{|c|c|c|}
\hline $\begin{array}{l}\text { 17. SECURITY CLASSIFICATION } \\
\text { OF REPORT }\end{array}$ & $\begin{array}{l}\text { 18. SECURTYY CLASSIFICATION } \\
\text { OF THIS PAGE }\end{array}$ & $\begin{array}{l}\text { 19. SECURTYY CLASSIFICATION } \\
\text { OF ABSTRACT }\end{array}$ \\
\hline Unclassified & Unclassified & Unclassified \\
\hline
\end{tabular}

15. NUMBER OF PAGES 17

16. PRICE CODE $\mathrm{AO3}$

20. LIMTTATION OF ABSTRACT

Unlimited 